\title{
Meteor-shower complex of asteroid 2003 EH1 compared with that of comet $96 \mathrm{P} / \mathrm{Machholz}{ }^{\star}$
}

\author{
L. Neslušan ${ }^{1}$, M. Hajduková, jr. ${ }^{2}$, and M. Jakubík ${ }^{1}$ \\ 1 Astronomical Institute, Slovak Academy of Science, 05960 Tatranská Lomnica, Slovakia \\ e-mail: [ne;mjakubik]@ta3.sk \\ 2 Astronomical Institute, Slovak Academy of Science, Dúbravská cesta 9, 84504 Bratislava, Slovakia \\ e-mail: astromia@savba.sk
}

Received 8 July 2013 / Accepted 27 September 2013

\section{ABSTRACT}

\begin{abstract}
Aims. We studied the structure of the meteoroid particle complexes released from asteroid 196256 (2003 EH1) to reveal the relationship to the meteor showers observed in Earth's atmosphere that belong to this complex as well. In addition, we studied the relationship between the asteroid and comet 96P/Machholz, which is situated in the same orbital phase space.

Methods. For nine perihelion passages of the parent asteroid in the past, we modeled the associated theoretical streams and followed their dynamical evolution until the present. Subsequently, we analyzed the orbital characteristics of the modeled streams, especially of the parts that approach Earth's orbit.

Results. We confirm the filamentary structure of the complex, which is qualitatively identical to the complex of 96P. Six wellestablished and two minor filaments approach the orbit of the Earth, producing four well-known meteor showers, daytime Arietids, Southern $\delta$-Aquarids, Quadrantids, and Northern $\delta$-Aquarids. The filaments corresponding to the Arietids and $\delta$-Aquarids $\mathrm{S}$ and $\mathrm{N}$ constitute the ecliptical component, and those corresponding to the Quadrantids and their southern counterpart constitute the toroidal component of the complex.
\end{abstract}

Key words. minor planets, asteroids: individual: 2003 EH1 - comets: individual: 96P/Machholz - meteorites, meteors, meteoroids

\section{Introduction}

The orbit of the parent body of the meteoroid stream is commonly situated within the orbital corridor of the respective stream. The Quadrantid meteoroid stream is an exception. The orbit of its originally suggested parent body (McIntosh 1990), comet 96P/Machholz, is situated clearly outside the corridor of the Quadrantids. Because of this circumstance and after the discovery of asteroid $2003 \mathrm{EH} 1$ in the orbit within the Quadrantid stream, the comet was, for a while, not thought to be the Quadrantid parent. Asteroid 196256 (hereafter 2003 EH1, the asteroid being well-known by this preliminary denotation) was regarded as the stream's sole parent (Jenniskens 2004), and the Quadrantids seemed to be the regular major stream, with the parent moving inside its corridor. However, more recent studies (Kaňuchová \& Neslušan 2007; Neslušan et al. 2013a) revealed that comet 96P may be the Quadrantid parent as well as 2003 EH1.

As noticed by McIntosh (1990), the orbital evolution of 96P, which has a quasi-periodical character, is similar to that of the Quadrantid stream, except that their 4000-year cycles are shifted by 2000 years. This similarity and the phase shift was also found in the orbital evolutions of 96P and asteroid $2003 \mathrm{EH} 1$ (Kaňuchová \& Neslušan 2007). Comet 96P has a meteor-shower complex that consists of several filaments that cross the Earth orbit (Neslušan et al. 2013a, hereafter NKT13), one of which corresponds to the Quadrantids. This means that it also corresponds to the orbit of $2003 \mathrm{EH} 1$, and we therefore expect that

* Tables 1 and 2 are available in electronic form at http: //www . aanda.org this asteroid is associated to a meteor-shower complex similar to that of $96 \mathrm{P}$.

The relationship between asteroid 2003 EH1 and the Quadrantids has been studied previously by several authors (Jenniskens 2004; Williams et al. 2004; Porubčan \& Kornoš 2005; Ryabova \& Nogami 2005; Wiegert \& Brown 2005; $\mathrm{Ki}-$ Won et al. 2009). An overall description of the whole complex, which consists of several meteor showers including major showers such as $\delta$-Aquarids and daytime Arietids, was recently published by Babadzhanov et al. (2008). Nevertheless, the authors proceeded in a different way than NKT13 and our current paper. They focused on the qualitative evolutionary features of the parent asteroid $2003 \mathrm{EH} 1$ and the associated meteoroid stream, which were revealed by numerically integrating the orbits of several representative clones.

We here describe the complex structure considering several models, each consisting of a large, statistically significant number of test particles that represent the meteoroids, and compare the structure with that of the sister complex of comet 96P.

As in NKT13, we use the words complex and stream in the meaning of synonyms referring to the whole structure of meteoroids released from the parent body. The complex consists of several filaments. If the particles of a filament hit the Earth, they can cause the meteor shower that corresponds to the filament.

\section{Modeling the stream dynamics}

To study the orbital evolution of test particles in the modeled theoretical stream, we followed the same procedure as in NKT13 (its Sect. 2). The method was initially suggested by 

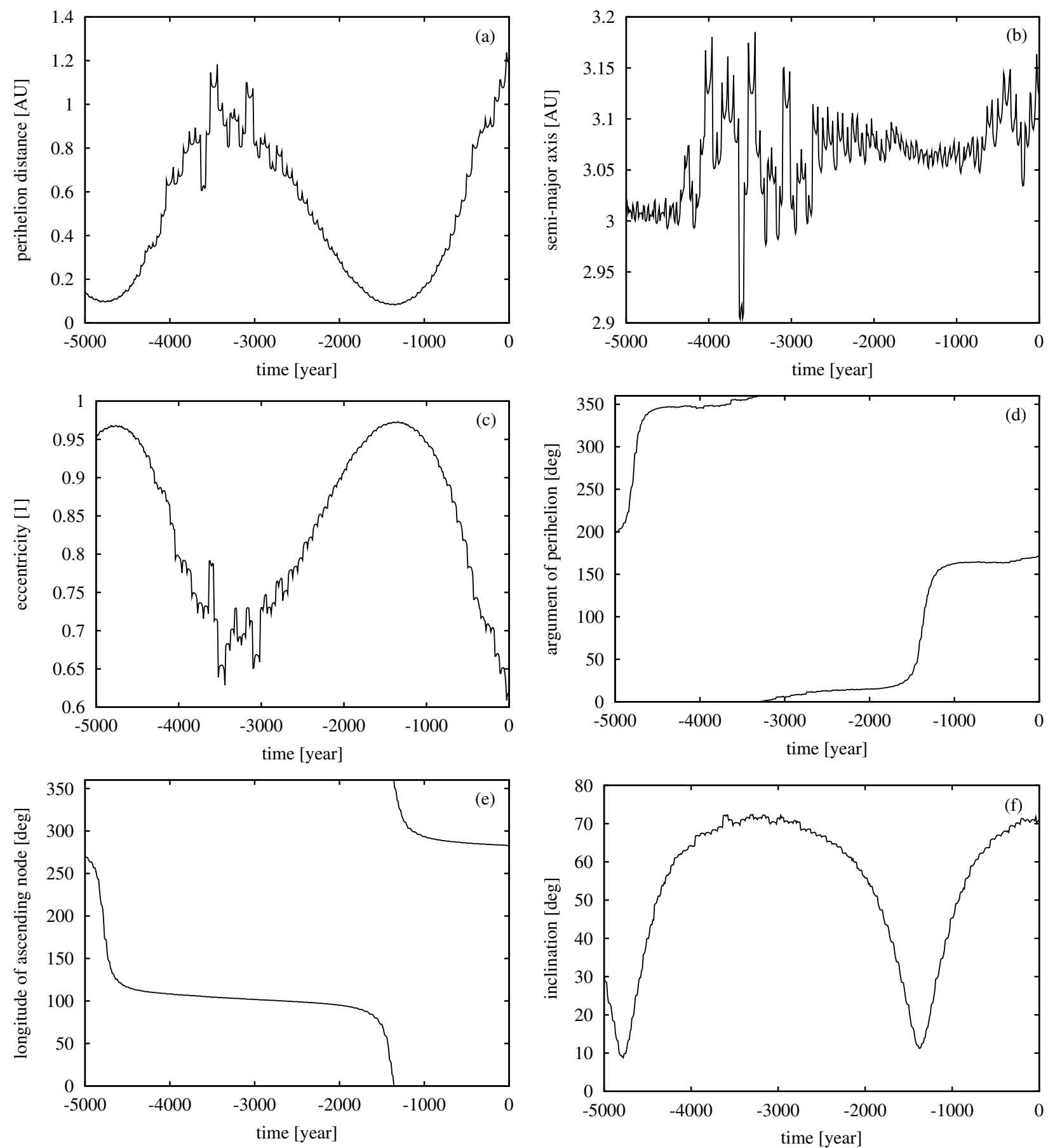

Fig. 1. Evolution of the perihelion distance a); semi-major axis b); eccentricity c); argument of perihelion d); longitude of ascending node e); and inclination to the ecliptic f); of the orbit of asteroid $2003 \mathrm{EH} 1$ during the past five millennia.

Neslušan (1999). Some improvements were added and the whole procedure is described in more detail in Tomko \& Neslušan (2012).

We recall that we use integrator RA15, which was developed by Everhart (1985) within the MERCURY package (Chambers 1999) to run the numerical integration. The gravitational perturbations of eight planets, from Mercury to Neptune, are considered. The initial orbital elements of the considered set of 10000 test particles, which represent the meteoroids, are calculated for the moment of the chosen parent perihelion passage in the past, whereby the radius vector of each particle is identical to the radius vector of the parent's orbit and the velocity vector is slightly changed by adding a small vector to the velocity vector of the parent. All these small velocity vectors correspond to an ejection of particles isotropically in all directions with uniform velocity, the size of which is arbitrarily chosen as one thousandth of the size of the parent's perihelion velocity.

Of course, the meteoroids are not ejected from the parent body only in its perihelion and with a uniform velocity. However, the purpose of our modeling is filling in the appropriate phase space of orbital elements, that is, creating the densest core of the stream. Apparently, this simple way of modeling in combination with a subsequent numerical integration of the particle orbits for a relaxation period produces the stream with essentially the same properties as a more sophisticated modeling. The relaxation 


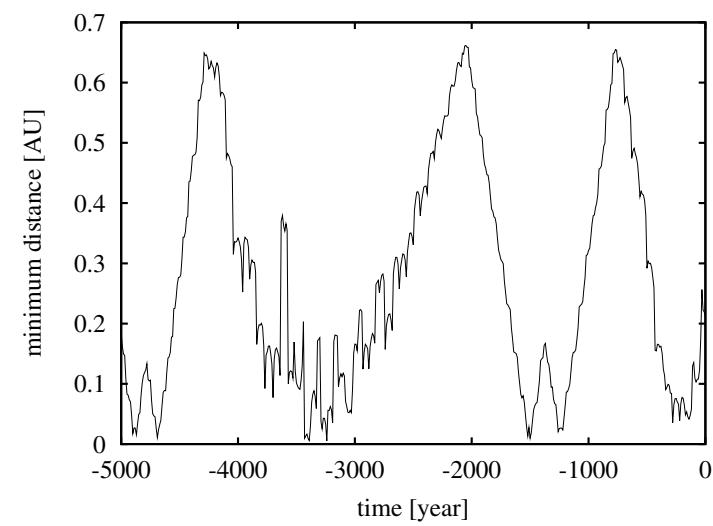

Fig. 2. Evolution of the minimum distance between the orbits of asteroid 2003 EH1 and Earth from 5000 years ago to the present.

period is always much shorter than the following dynamical evolution of the stream. The numerical integration is, then, continuously extended until the present. At its end, we selected all particles in orbits that closely approach Earth's orbit (within $0.05 \mathrm{AU})$. The dynamical characteristics of these particles are similar to those of corresponding showers and, therefore, we used them to predict the showers.

If there were enough particles to predict a shower, we tried to identify the Earth-orbit approaching particles with the actually observed meteors. In this identification, the photographic IAU MDC (Lindblad et al. 2003), radio-meteor (Hawkins 1963; Sekanina \& Southworth 1975; Lindblad, 2003, priv. comm.), and the SonotaCo video-meteor databases (SonotaCo 2009) were used. To identify a predicted shower to its potential counterpart in a given database, we used the break-point method (Neslušan et al. 2005, 2013b).

We modeled all theoretical streams in the perihelia of the nominal orbit of the parent asteroid. The past evolution of the individual elements of this orbit is shown in Fig. 1. The nominal orbit of 2003 EH1 was downloaded from the JPL Small-Body Database Browser ${ }^{1}$ on October 9, 2012. The orbital elements are $q=1.1898208 \mathrm{AU}, e=0.6190588, \omega=171.3249^{\circ}$, $\Omega=282.95809^{\circ}, i=70.87006^{\circ}$, and $T(\mathrm{JD})=2456729.40538$ for epoch 2456200.5 .

In Fig. 2, we show the evolution of the minimum distance between the orbit of $2003 \mathrm{EH} 1$ and the orbit of Earth. We see that the asteroid passed Earth's orbit closely (within $0.1 \mathrm{AU}$ ) in several short periods. For the rest, the orbits of 2003 EH1 and Earth were quite distant. We note that the dynamics of $2003 \mathrm{EH} 1$ is obviously controlled by Jupiter. This claim is supported by a negligible change of the Tisserand parameter calculated with respect to this planet. During five millennia of studied evolution of $2003 \mathrm{EH} 1$ nominal orbit, the parameter varies only from 2.038 to 2.122 .

For $96 \mathrm{P}$ as for $2003 \mathrm{EH} 1$, the orbital evolution of most of the considered test particles has the same features as the evolution of the parent's orbit itself, except for the phase that can be shifted about the entire cycle of the quasi-periodic variation. One circulation period of the longitude of the ascending node and, at the same time, the argument of perihelion lasts 8.2 millennia for 2003 EH1 as well.

Similar to 96P, the nodes of the orbit of 2003 EH1 cross Earth's orbit at eight points during its evolutionary cycle (Babadzhanov \& Obrubov 1992; Babadzhanov et al. 2008).

\footnotetext{
1 http://ssd.jpl.nasa.gov/sbdb.cgi
}

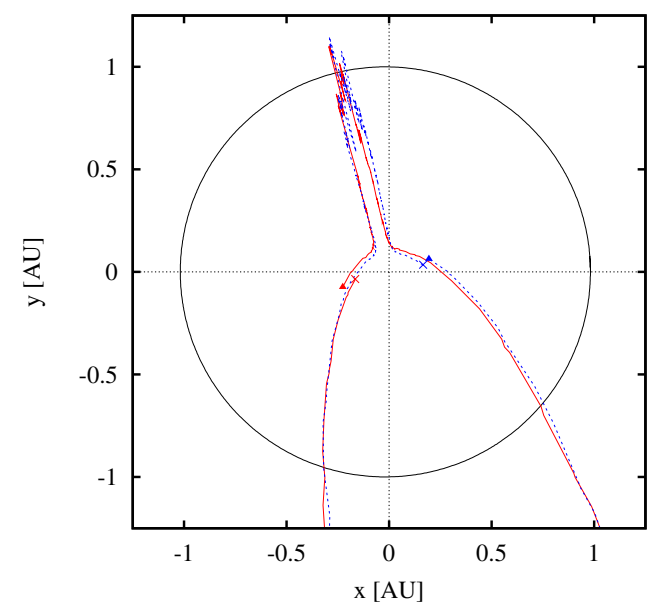

Fig. 3. Evolution of the orbital node position of asteroid $2003 \mathrm{EH} 1$ during one circulation period of the longitude of the ascending node (which is the same as the circulation period of the argument of perihelion). The solid red (dashed blue) curve shows the shift in the descending (ascending) node, which corresponds to the radiant northward (southward) of the ecliptic. The crosses indicate the starting positions and the full triangles the final positions of the nodes in the considered circulation period.

The evolution of the cross-point position for one period of the $\Omega$-circulation is illustrated in Fig. 3 . The solid red curve illustrates the shift in the descending node (the radiant of corresponding meteors is situated northward of the ecliptic) and the dashed blue curve plots the shift in the ascending node (the radiant is southward of the ecliptic) of the asteroid orbit. We can see that each node basically crosses Earth's orbit four times, twice at the outward and twice at the inward shift.

The evolution of the position of both nodes was described in NKT13 previously for 96P. Nevertheless, we recall the description, which is analogous, for $2003 \mathrm{EH} 1$. The above-mentioned narrow loop related to the descending node (the solid red curve in Fig. 3) corresponds to the well-known Quadrantid meteor shower. Although Babadzhanov \& Obrubov (1992) assigned the cross-point of the outward-shifting part of the curve to the Quadrantids and the inward shifting part to another shower, the Ursids, we find that this conceptual resolution is impossible in a quantitative study of a real or theoretical Quadrantid stream. For a large number of meteoroids, the interval of the cross-points related to the nodes that shift outward is almost entirely overlapped by the interval of those cross-points that are related to the nodes that shift inward. The orbital parameters and radiants corresponding to meteoroids in these intervals also lie in the appropriate overlapping intervals. One has no serious quantitative criterion for distinguishing the two showers. The same conclusion can be drawn for the narrow loop related to the ascending node (the dashed blue curve in Fig. 3), therefore we assigned the two cross-sections of the given narrow loop only to a single northern and single southern meteor shower. Thus, we predict that the complex of $2003 \mathrm{EH} 1$ causes six meteor showers that are observable in Earth's atmosphere.

\section{Predicted showers}

Because of reasons introduced in NKT13, we modeled the streams at the moments of several parent-body perihelion passages in the past. To keep a certain consistency, we chose essentially the same times of modeling as were chosen by NKT13 for comet 96P. 

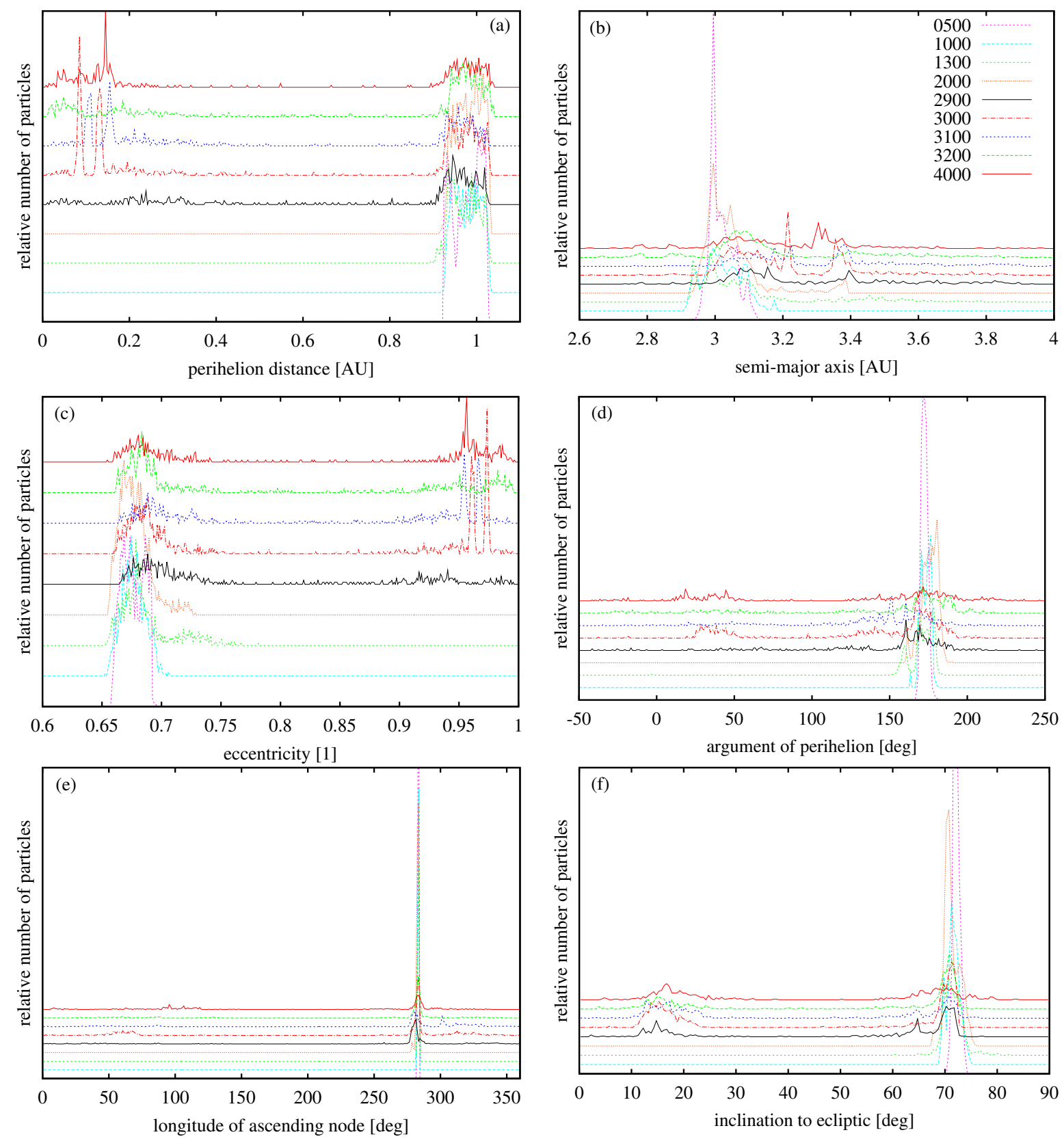

Fig. 4. Distributions of elements of the modeled particle orbits approaching Earth's orbit at a distance shorter than 0.05 AU at present. Specifically, relative distributions, in arbitrary units, of the perihelion distance a), semi-major axis b); eccentricity c); argument of perihelion d); longitude of ascending node e); and inclination to the ecliptic f) are shown for nine streams modeled for nine times before the present. The times are indicated at the right top corner of plot b). For clarity, the distribution for each successive time is shifted up relatively to the distribution for the preceding time and only two thirds of the peak in the distribution of the longitude of the ascending node and inclination (e) and f)) for model M500 are shown in the chosen vertical scale.

Specifically, we modeled the streams in times corresponding to $2003 \mathrm{EH} 1$ 's perihelion passages about 500, 1000, 1300, 2000, 2900, 3000, 3100, 3200, and 4000 years ago. Hereafter, we refer to these models for instance as M500 (modeling for a time 500 years ago). The times of 500, 1000, 2000, 3000, and 4000 years were chosen arbitrarily to cover the entire expected interval for the evolution of most part of the meteoroids in the current stream. To see some specific aspects of the evolution on the timescale of a century, we also modeled the stream at times 2900 (3000 is also used in this context), 3100, and 3200 years before the present. Because the ejection rate of meteoroid particles from the parent body is assumed to be proportional to the distance from the Sun, we can expect the highest rate in the period, in which the parent's perihelion distance is at the minimum of its quasi-periodical variation. For $2003 \mathrm{EH} 1$, this occurred about 1300 years ago (see Fig. 1a). Therefore, we also modeled a stream for the time of 1300 years before the present.

The size of the uniform ejection velocity of the test particles was chosen to be $0.001 v_{\mathrm{pp}}$, where $v_{\mathrm{pp}}$ is the perihelion velocity of $2003 \mathrm{EH} 1$ in the actual model. Accordingly, the specific values of this velocity are $52.39,103.14,141.76,75.90,28.85$, $36.27,31.85,43.09$, and $126.41 \mathrm{~m} \mathrm{~s}^{-1}$ in the models from M500 to M4000, respectively.

Apparently, a significant part of the modeled particles evolve into the orbits that approach Earth's orbit within the chosen limit of $0.05 \mathrm{AU}$ (the use of this value was discussed in NKT13). For 

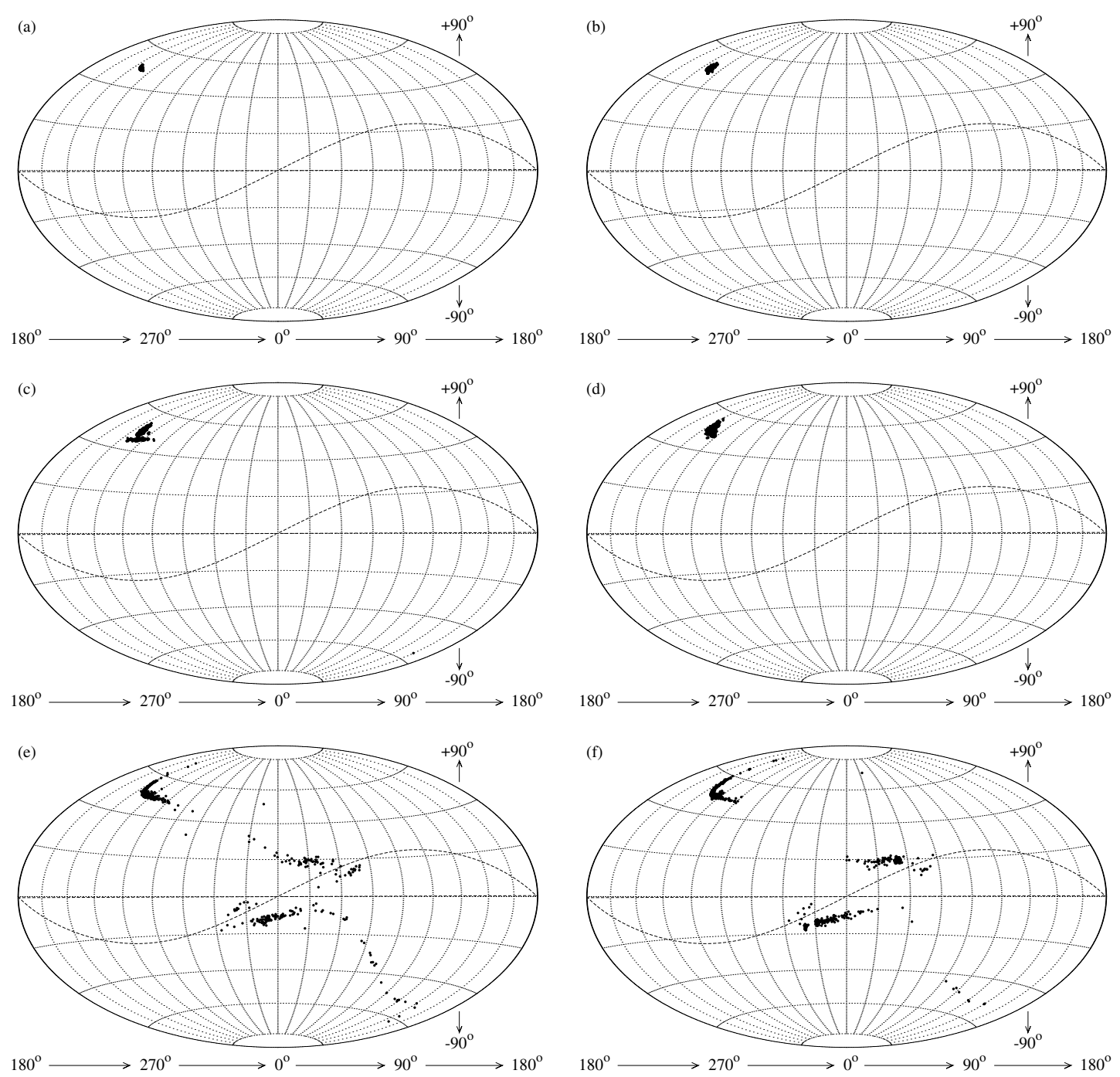

Fig. 5. Positions of radiants of the 2003 EH1-stream particles moving currently in orbits where they can approach Earth's orbit within 0.05 AU. The positions for streams M500, M1000, M1300, M2000, M2900, M3000, M3100, M3200, and M4000 are shown in plots a)-i), respectively. (Plots $\mathbf{g})-\mathbf{i}$ ) are in the second part of the figure on the next page.) The stream is split into eight filaments with eight distinct radiant areas. The enumeration of the filaments is given in plot i) with the complete set of filaments (F1 to F8). The radiants are shown in the Hammer projection of the sky. The equatorial coordinate frame is used with the right ascension indicated by meridional circles and the declination is indicated with the declination circles. The sinusoid-like curve illustrates the ecliptic.

these particles, we predict the theoretical showers that are expected to be observed in Earth's atmosphere if 2003 EH1 is the active source of meteoroids.

There are six filaments of the stream that were previously identified in NKT3 in the meteoroid complex of comet 96P, which approach Earth's orbit. In addition, the complex of 2003 EH1 consists of another two filaments, which we refer to as filaments 7 and 8 . The radiants of particles in filaments $1-3$ and 7 are situated northward of the ecliptic, and in filaments 4-6 and 8 they are located southward of this plane. In other words, the former collide with Earth at their descending and the latter at their ascending node.

In analogy with NKT13, we divided the part of each modeled stream that approached Earth into the filaments on the basis of the radiant position of meteors. The members of a given filament have a common radiant area. The radiant areas of the individual filaments are delimited by the following conditions:

filament $1: 10^{\circ}<\alpha_{\mathrm{g}}<50^{\circ}$ and $\delta_{\mathrm{g}}<30^{\circ}$;

filament 2: $\alpha_{\mathrm{g}}>325^{\circ}$ or $\alpha_{\mathrm{g}}<5^{\circ}$;

filament 3: $200^{\circ}<\alpha_{\mathrm{g}}<265^{\circ}$ and $40^{\circ}<\delta_{\mathrm{g}}<60^{\circ}$; filament 4: $30^{\circ}<\alpha_{\mathrm{g}}<60^{\circ}$;

filament 5: $\alpha_{\mathrm{g}}>320^{\circ}$ or $\alpha_{\mathrm{g}}<20^{\circ}$;

filament 6: $100^{\circ}<\alpha_{\mathrm{g}}<160^{\circ}$ and $-60^{\circ}<\delta_{\mathrm{g}}<-45^{\circ}$;

filament 7: $80^{\circ}<\alpha_{\mathrm{g}}<100^{\circ}$ and $\delta_{\mathrm{g}}<40^{\circ}$;

filament 8: $80^{\circ}<\alpha_{\mathrm{g}}<100^{\circ}$ and $\delta_{\mathrm{g}}>10^{\circ}$;

where $\alpha_{\mathrm{g}}$ and $\delta_{\mathrm{g}}$ are the geocentric coordinates. In addition, the radiants of filaments $1-3$, and 7 (filaments 4-6, and 8) have to obey the condition of descending (ascending) node, therefore their ecliptical latitude has to be $\beta_{\mathrm{g}}>0^{\circ}\left(\beta_{\mathrm{g}}<0^{\circ}\right)$.

The obtained mean geophysical characteristics of all eight filaments, in the models where they exist, are given in Table 1 and the mean orbital elements in Table 2. The positions of the radiants on the sky of all particles in the orbits that approach that of Earth within 0.05 AU are shown in Fig. 5. In addition to the more or less compact radiant areas of the filaments, we can see in this figure the radiants of some particles in transit orbits between the filaments. A bridge that corresponds to the transit between filaments 5 (Southern $\delta$-Aquarids) and 6 is seen especially in models M2900, M3000, and M3200 (Figs. 5e, g, and h). An indication of the transit between filaments 1 (Arietids) and 

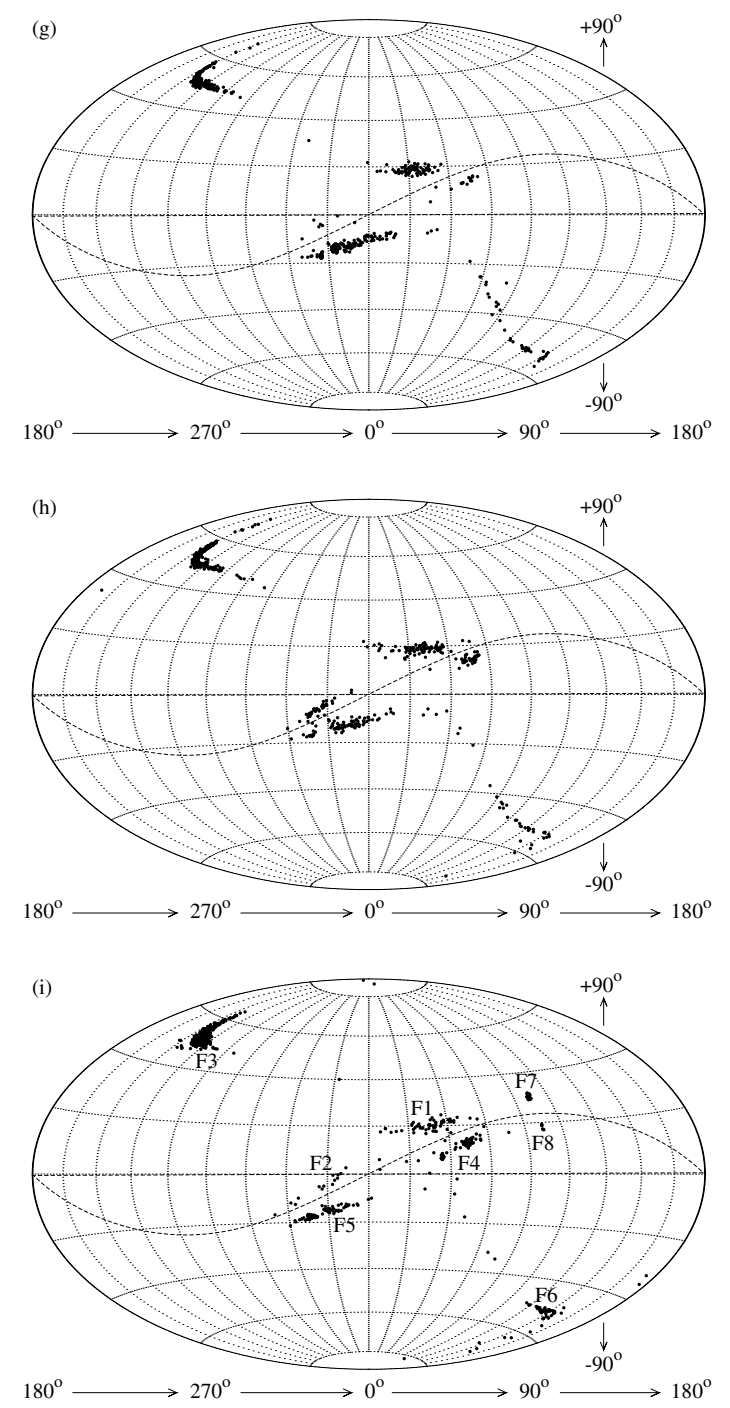

Fig. 5. continued.

3 (Quadrantids) is seen in model M2900 (Fig. 5e). The specific features of the individual filaments are described in the following subsections.

\subsection{Filaments corresponding to the well-known showers}

In the complex of $96 \mathrm{P}$ as well as in the complex of 2003 EH1 studied here, filaments 1, 2, 5, and 3 correspond to the well-known, major meteor showers: the daytime Arietids, the Northern and Southern $\delta$-Aquarids, and the Quadrantids, respectively. Because we have quite a rich sample of observational data for these showers, comparing the prediction for showers originating from both parent bodies, comet $96 \mathrm{P}$ and asteroid $2003 \mathrm{EH} 1$, might help us, in principle, to answer the question which of these two bodies is the dominant or, possibly, exclusive source of the complex meteoroids. The actual comparison does not enable us to draw a decisive conclusion, however. There is a certain uncertainty in our prediction of the mean parameters of individual filaments due to the differences among the nine models. The uncertainty does not allow a strict comparison of the prediction with the observational data. Within the uncertainty, the prediction using the models of the 96P complex is about as good as that using the models of the complex of $2003 \mathrm{EH} 1$.

As expected, a richer Quadrantid shower is predicted in the shower originating from $2003 \mathrm{EH} 1$ than for that originating from
96P, because the current orbit of the asteroid is in the same evolutionary phase as that of this shower, while the orbit of the comet is shifted by about a quarter of the quasi-period cycle of its evolution. Unfortunately, the observational data were often gained within some observational campaigns and are therefore not homogeneous. Thus, we do not know the relative abundances of the Quadrantids and the Southern $\delta$-Aquarids, for instance, which are the second-most abundant night showers of the complex.

We can state that the above-mentioned four showers can originate either from comet $96 \mathrm{P}$ or from asteroid $2003 \mathrm{EH} 1$, or from both these parents.

\subsection{Filaments 4 and 6 without observed counterparts}

Filament 4 is predicted to be the southern branch of the daytime Arietids. In the considered radio-meteor database, we identified a shower with the geocentric radiant $\alpha_{\mathrm{g}}=4.1^{\circ}, \delta_{\mathrm{g}}=+10.1^{\circ}$ and geocentric velocity $V_{\mathrm{g}}=35.7 \mathrm{~km} \mathrm{~s}^{-1}$, which resembles this filament. Nevertheless, the real shower, in comparison with filament 4 , clearly has the lower geocentric velocity (cf. 35.7 to $38.6-42.8 \mathrm{~km} \mathrm{~s}^{-1}$ ) and semi-major axis (cf. $1.75 \mathrm{AU}$ to the predicted value of 2.9 to $3.0 \mathrm{AU}$ for filament 4 ) as well as a slightly higher inclination, $i \sim 22.0^{\circ}$, (cf. the predicted values ranging from $16.3^{\circ}$ to $19.3^{\circ}$ ). In addition, the real shower is not very numerous, consisting of only 11 radio meteors. Therefore, one has to be careful in identifying the filament with the real shower. The perihelion distance of filament 4 is predicted to be the shortest in the complex, together with that of $\delta$-Aquarids $\mathrm{N}$. Therefore, the lifetime of the particles in the corresponding shower is expected to be short.

In model M4000, the regular filament 4 is denoted $b$-filament, because there is another low-abundant shower with the same radiant area, the $a$-filament (see Tables 1 and 2), which cannot be identified even with a very diffuse real shower. We list it to point out this alternative dynamical possibility according to the theory.

Filament 6 cannot be identified with a corresponding shower in the considered databases. The reason is, most probably, the very low number of meteors with the radiant on the southern sky. The discussion about the relationship of filament 6 with real showers was published in NKT13.

\subsection{New filaments 7 and 8}

In model M4000 of the 2003 EH1 complex, there occurred a shower that had both northern and southern branches, which we refer to as filaments 7 and 8, respectively. The shower, if it exists, is expected to be active for a few days in the middle of July on the day sky. This is the ecliptical shower related to the Arietids. This relationship is apparent when the positions of radiants are plotted in the ecliptical coordinate frame with the longitude shifted in such a way that Earth's apex is at the origin of the frame (Fig. 6; see also the discussion in Sect. 4). Then the radiant area of filament 7 is shifted to the radiant area of the Arietids (filament 1) and the radiant area of filament 8 to that of filament 4 which is regarded as the southern branch of the Arietids. (This is the reason why the radiant areas of filaments 7 and 8 cannot be distinguished from those of filaments 1 and 4 in Fig. 6.)

Filaments 7 and 8 were not predicted at the complex of 96P. This circumstance might help us, in principle, to decide whether the asteroid $2003 \mathrm{EH} 1$ was an active source of meteoroids several millennia ago. Namely, if the real counterparts of 


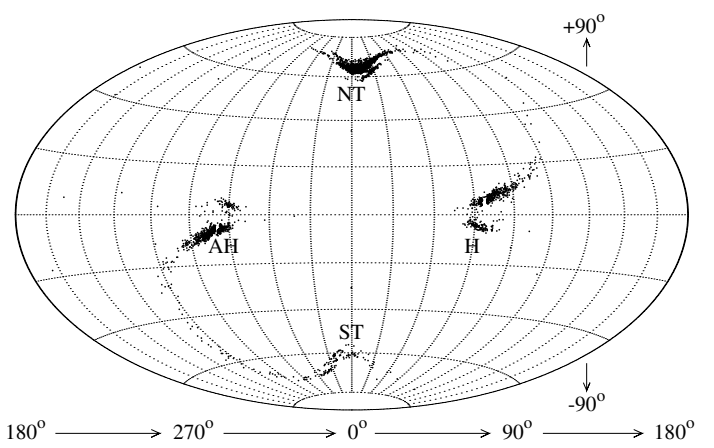

Fig. 6. Ecliptic-toroidal structure of the meteoroid complex of asteroid $2003 \mathrm{EH} 1$. The plot illustrates the positions of radiants in the modified ecliptical coordinate frame $\left(\lambda_{\mathrm{A}}, \beta_{\mathrm{g}}\right)$ in the Hammer projection of the celestial sphere. The common ecliptic longitude, $\lambda_{\mathrm{g}}$, is replaced with the angle $\lambda_{\mathrm{A}}$ measured from the ecliptic longitude of Earth's apex. This apex is situated at the beginning of the frame. Abbreviations NT, ST, H, and $\mathrm{AH}$ stand for the northern toroidal, southern toroidal, helion, and antihelion directions, respectively (see text of Sect. 4).

filaments 7 and 8 existed, they could have originated only from the asteroid. Conversely, if we proved that the counterparts do not exist, the asteroid would have to be an inactive body in the past.

Because the shower is predicted to occur at daytime, with the mean radiant in the angular distance only $19^{\circ}$ to $22^{\circ}$ from the Sun at the maximum activity, we can find it only in the radio-meteor data. Unfortunately, the result of the search is highly disputable. We employed the break-point method used for selecting meteors of the suspected shower from the database. To do this, we constructed the dependence of the number of selected meteors on the threshold value of the $D$-discriminant. In this dependence, there occurred a mild peak for $D=0.16$ for filament 7 . Within this limit, we selected 20 meteors with the mean $q, e, \omega, \Omega$, and $i$ equal to $0.134 \mathrm{AU}, 0.921,34.1^{\circ}, 121.5^{\circ}$, and $17.2^{\circ}$, respectively. At $D=0.18$, the number of selected meteors sharply increased, but this was because of the selection of members of another shower, which was clearly not related to filament 7 .

Because the number of selected meteors corresponding to filament 7 was low and the break point was scarcely recognizable, we confirmed a shower in the radio database with the method suggested by Neslušan et al. (2013b, Sect. 4). A standard threshold value of $D$ equal to 0.20 is suggested to be used in the method. Because the acceptable value of $D$ is lower than 0.18 in our case and we have an indication of an actual break point in $D=0.16$, we considered this last value instead of 0.20 . Starting the iteration procedure in the break-point method from initial orbits with each parameters incremented (decremented) to about twice of its determination error, $\sigma$, we can state one success and one failure in the convergence of the iteration to the first above-mentioned mean orbit. Specifically, when we started from the "plus $2 \sigma$ orbit" with $q=0.234 \mathrm{AU}, e=1.011, \omega=53.3^{\circ}$, $\Omega=142.3^{\circ}$, and $i=26.4^{\circ}$, the iteration converged to the orbit characterized with $q=0.154 \mathrm{AU}, e=0.893, \omega=36.2^{\circ}$, $\Omega=155.1^{\circ}$, and $i=18.1^{\circ}$. This means that there is a trend toward the originally found orbit of the shower (although the trend is not confirmed in $\Omega$ ). However, when we started from the "minus $2 \sigma$ orbit" with $q=0.034 \mathrm{AU}, e=0.831, \omega=14.9^{\circ}$, $\Omega=100.7^{\circ}$, and $i=8.0^{\circ}$, the iteration converged to a clearly different orbit with the elements $q=0.207 \mathrm{AU}, e=0.854$, $\omega=153.8^{\circ}, \Omega=207.4^{\circ}$, and $i=4.9^{\circ}$.

A similar analysis made for filament 8 did not reveal a real shower. Since this filament is predicted to be an order of magnitude less abundant than filament 7 (see the last column in Table 1), the negative result is no surprise.

Clearly, the partial success in the case of filament 7 motivates us to search for a real shower corresponding to this filament in a much richer database of radio meteors.

\section{Ecliptic-toroidal structure}

The parts of meteoroid complexes that approach Earth's orbit of comet 96P (NKT13) and asteroid 2003 EH1 are arranged in an ecliptic-toroidal structure. This means that the meteors constituting these complexes approach Earth with a significantly higher rate from the cardinal directions on the sky. The ecliptic-toroidal structure of the $2003 \mathrm{EH} 1$ complex can be seen in Fig. 6. The radiants are plotted in ecliptic coordinates $\lambda_{\mathrm{g}}$ and $\beta_{\mathrm{g}}$ with the ecliptical longitude, $\lambda_{\mathrm{g}}$, shifted in such a way that the apex of Earth's motion is at the origin of the coordinate system, that means $\lambda_{\mathrm{g}}$ is replaced with $\lambda_{\mathrm{A}}=\lambda_{\mathrm{g}}-\left(\lambda_{\odot}+270^{\circ}\right)$, where $\lambda_{\odot}$ is the ecliptical longitude of the Sun at the time of the meteor fall.

The ecliptical showers of the $2003 \mathrm{EH} 1$ complex were predicted with the help of filaments $1,2,4,5,7$, and 8 and their particles come from the direction toward the Sun named helion and the opposite direction named antihelion. We note that the radiant areas of filaments 7 and 8 appear to be inside those of filaments 1 and 4 , respectively, when the coordinates $\lambda_{\mathrm{A}}$ and $\beta_{\mathrm{g}}$ were used to plot the radiants. The particles in filaments 3 and 6 come from the northern and southern toroidal directions, which are known especially from the radar observations of meteors (e.g. Campbell-Brown \& Brown, 2005).

We can state that, after comet 96P/Machholz, $2003 \mathrm{EH} 1$ is another parent body associated with more than one single meteor shower (if it actually is a significant source of meteoroid particles). As expected, the structure of its meteoroid complex is similar to that of $96 \mathrm{P}$ also in sense of the cardinal meteor directions.

\section{Conclusions}

Our analysis of the dynamics of meteoroid particles assumed to be released from the surface of near-Earth asteroid 196256 (2003 EH1) revealed the complicated structure of its meteoroid stream, which approaches Earth's orbit in eight filaments. Four of these filaments correspond to the well-known meteor showers, daytime Arietids, the Southern and Northern $\delta$ Aquarids, and the Quadrantids.

In the context of the cardinal meteor directions, the complex consists of both types of showers: ecliptical (helion and antihelion) and toroidal. Of two toroidal showers the northern, Quadrantid, shower is real and the southern shower is hypothetical at the moment. However, there is a chance that the shower might soon be discovered in the photographic data produced by the newly installed fireball network in southwest Australia (Bland et al. 2005, 2008).

The meteoroid complex of $2003 \mathrm{EH} 1$ is qualitatively almost identical to the complex of comet 96P/Machholz. The difference are two additional filaments, here numbered as filaments 7 and 8 , which occurred only in one of nine models of the $2003 \mathrm{EH} 1$ stream (model M4000), however. In fact, the complex can be regarded as a unique structure originating, most likely, in two parent bodies, one cometary and one asteroidal.

Since there are several tens of Jupiter-family comets, many of which have dynamical properties similar to those of $96 \mathrm{P}$ and $2003 \mathrm{EH} 1$, and several known asteroids with their perihelion in 
the interior of Earth's orbit, the meteoroid complex of 96P-2003 $\mathrm{EH} 1$ can be the prototype of an entire group of such complexes. Discovering and studying them is a challenge for future meteor research.

Acknowledgements. This article was supported by the realization of Project ITMS No. 26220120029, based on the supporting operational Research and development program financed by the European Regional Development Fund. The work was also supported, in part, by VEGA - the Slovak Grant Agency for Science, grant No. 0011 and by the Slovak Research and Development Agency under contracts Nos. APVV-0158-11 and APVV-0517-12.

\section{References}

Babadzhanov, P. B., \& Obrubov, Y. V. 1992, Solar System Research, 26, 288 Babadzhanov, P. B., Williams I. P., \& Kokhirova, G. I. 2008, MNRAS, 386, 227 Bland, P. A., Spurný, P., Bevan, A. W. R., et al. 2005, Meteor. Planet. Sci. Suppl., 40,5225

Bland, P. A., Spurný, P., Shrbený, L., et al. 2008, LPI Contribution, 1405, 8246 Campbell-Brown, M. D., \& Brown, P. 2005, LPI Contribution, 1280, 29 Chambers, J. E. 1999, MNRAS, 304, 793

Chernitsov, A. M., Baturin, A. P., \& Tamarov V. A. 1998, Sol. Syst. Res., 32, 405

Everhart, E. 1985, in Dynamics of Comets: Their Origin and Evolution, eds. A. Carusi, \& G. B. Valsecchi (Dordrecht: Kluwer) 185
Hawkins, G. S. 1963, Smithsonian Contrib. Astrophys., 7, 53

Jenniskens, P. 2004, AJ, 127, 3018

Kaňuchová, Z., \& Neslušan, L. 2007, A\&A, 470, 1123

Ki-Won, L., Hong-Jin, Y., \& Myeong-Gu, P. 2009, MNRAS, 400, 1389

Lindblad, B. A., Neslušan, L., Porubčan, V., \& Svoreň, J. 2003, Earth Moon Planets, 93, 249

McIntosh, B. A. 1990, Icarus, 86, 299

Milani, A. 1999, Icarus, 137, 269

Milani, A., Sansaturio, M. E., Tommei, G., Arratia, O., \& Chesley, S. R. 2005, A\&A, 431, 729

Neslušan, L. 1999, A\&A, 351, 752

Neslušan, L., Svoreň, J., \& Porubčan, V. 1995, Earth Moon Planets, 68, 427

Neslušan, L., Kaňuchová, Z., \& Tomko, D. 2013a, A\&A, 551, A87 (NKT13)

Neslušan, L., Svoreň, J., \& Porubčan, V. 2013b, Earth Moon Planets, 110, 41

Porubčan, V., \& Kornoš, L. 2005, Contrib. Astron. Obs. Skalnaté Pleso, 35, 5

Ryabova, G., \& Nogami, N. 2005, in Proc. Internat. Meteor Conference, Varna, Bulgaria, eds. M. Triglav-Čekada, J. Kac, \& A. McBeath (International Meteor Organization), 63

Sekanina, Z., \& Southworth, R. B. 1975, Physical and dynamical studies of meteors. Meteor-fragmentation and stream-distribution studies (Cambridge, MA: Final Report Smithsonian Astrophys. Obs)

SonotaCo. 2009, Journal of the IMO, 37, 55 (http://sonotaco.jp/doc/ $\mathrm{SNM} /$ )

Tomko, D., \& Neslušan, L. 2012, Earth Moon Planets, 108, 123

Wiegert, P., \& Brown P. 2005, Icarus, 179, 139

Williams, I. P., Ryabova, G. O., Baturin, A. P., \& Chernitsov, A. M. 2004, MNRAS, 355, 1171 
Table 1. Mean geophysical characteristics of the predicted meteor showers.

\begin{tabular}{|c|c|c|c|c|c|c|c|c|}
\hline Shower & $t_{\mathrm{evol}}$ & $t_{\text {act }}$ or D.B. & $\alpha_{\mathrm{g}}$ & $\delta_{\mathrm{g}}$ & $V_{\mathrm{g}}$ & $V_{h}$ & $\gamma$ & $A$ or $N_{\text {sel }}$ \\
\hline \multirow[t]{5}{*}{ First } & 2900 & May 7-May 15 & $20.4 \pm 4.8$ & $+18.6 \pm 1.6$ & $33.3 \pm 2.3$ & $39.0 \pm 0.3$ & 18.0 & 0.0038 \\
\hline & 3000 & May 7-May 27 & $33.3 \pm 2.5$ & $+19.3 \pm 1.0$ & $39.1 \pm 2.6$ & $38.2 \pm 1.3$ & 23.7 & 0.0235 \\
\hline & 3100 & Apr. 16-May 17 & $22.6 \pm 4.7$ & $+18.7 \pm 1.3$ & $34.4 \pm 2.4$ & $38.9 \pm 0.4$ & 17.2 & 0.0069 \\
\hline & 3200 & Apr. 19-June 3 & $27.8 \pm 6.7$ & $+18.8 \pm 1.4$ & $36.4 \pm 4.1$ & $38.3 \pm 1.8$ & 22.1 & 0.0080 \\
\hline & 4000 & May 2-June 10 & $33.0 \pm 7.0$ & $+20.3 \pm 1.8$ & $39.0 \pm 3.9$ & $38.4 \pm 1.4$ & 24.7 & 0.0048 \\
\hline \multirow[t]{7}{*}{ Second } & 2900 & July 17-Aug. 5 & $335.1 \pm 3.4$ & $-6.0 \pm 2.1$ & $42.9 \pm 3.2$ & $37.9 \pm 3.0$ & 150.3 & 0.0010 \\
\hline & 3000 & July 19-Aug. 1 & $334.4 \pm 3.8$ & $-6.2 \pm 2.0$ & $41.9 \pm 3.0$ & $37.2 \pm 1.8$ & 148.0 & 0.0004 \\
\hline & 3100 & July 17-Aug. 14 & $336.7 \pm 6.2$ & $-4.7 \pm 3.4$ & $40.6 \pm 2.3$ & $37.0 \pm 1.5$ & 149.4 & 0.0005 \\
\hline & 3200 & July 15-Aug. 10 & $336.4 \pm 5.4$ & $-5.9 \pm 2.9$ & $41.8 \pm 3.8$ & $37.5 \pm 2.6$ & 151.8 & 0.0037 \\
\hline & 4000 & July $13-$ Aug. 18 & $338.5 \pm 9.1$ & $-4.4 \pm 4.7$ & $41.4 \pm 3.2$ & $38.0 \pm 1.9$ & 153.6 & 0.0013 \\
\hline & $\mathrm{F}$ & July 30-Aug. 16 & $342.9 \pm 3.5$ & $-0.4 \pm 2.8$ & $39.6 \pm 2.5$ & $37.0 \pm 2.0$ & & 18 \\
\hline & $\mathrm{V}$ & Aug. 6-Aug. 18 & $345.9 \pm 2.5$ & $+1.3 \pm 1.7$ & $39.1 \pm 1.6$ & $36.6 \pm 1.5$ & & 36 \\
\hline \multirow[t]{11}{*}{ Third } & 500 & Jan. 3-Jan. 4 & $229.5 \pm 1.3$ & $+49.5 \pm 0.4$ & $41.3 \pm 0.3$ & $38.9 \pm 0.1$ & 87.1 & 0.2053 \\
\hline & 1000 & Jan. 3-Jan. 5 & $229.4 \pm 1.4$ & $+50.0 \pm 1.0$ & $41.1 \pm 0.6$ & $38.9 \pm 0.1$ & 87.4 & 0.1334 \\
\hline & 1300 & Jan. 3-Jan. 5 & $229.4 \pm 1.5$ & $+50.2 \pm 1.5$ & $41.0 \pm 0.7$ & $38.9 \pm 0.1$ & 87.6 & 0.0773 \\
\hline & 2000 & Jan. 2-Jan. 5 & $228.1 \pm 2.4$ & $+50.9 \pm 1.3$ & $40.9 \pm 0.7$ & $38.9 \pm 0.1$ & 88.6 & 0.1791 \\
\hline & 2900 & Dec. 25-Jan. 9 & $231.9 \pm 6.7$ & $+51.0 \pm 1.8$ & $39.9 \pm 1.4$ & $39.1 \pm 0.2$ & 86.0 & 0.0418 \\
\hline & 3000 & Dec. 25-Jan. 9 & $229.9 \pm 4.9$ & $+50.9 \pm 2.1$ & $40.5 \pm 0.8$ & $39.0 \pm 0.1$ & 87.5 & 0.0657 \\
\hline & 3100 & Dec. 27-Jan. 12 & $232.2 \pm 6.9$ & $+50.8 \pm 1.9$ & $39.9 \pm 1.5$ & $39.1 \pm 0.2$ & 85.9 & 0.0299 \\
\hline & 3200 & Dec. 27-Jan. 9 & $228.4 \pm 4.2$ & $+51.5 \pm 2.3$ & $40.6 \pm 0.6$ & $38.9 \pm 0.1$ & 88.7 & 0.0485 \\
\hline & 4000 & Dec. 29-Jan. 8 & $230.5 \pm 5.0$ & $+52.3 \pm 2.8$ & $39.6 \pm 1.4$ & $38.9 \pm 0.1$ & 88.5 & 0.0237 \\
\hline & $\mathrm{F}$ & Jan. 2-Jan. 8 & $230.4 \pm 2.2$ & $+49.2 \pm 1.0$ & $41.0 \pm 1.0$ & $38.8 \pm 0.6$ & & 59 \\
\hline & $\mathrm{V}$ & Jan. 1-Jan. 8 & $230.0 \pm 1.7$ & $+49.5 \pm 1.0$ & $40.2 \pm 0.8$ & $38.2 \pm 0.4$ & & 423 \\
\hline \multirow[t]{6}{*}{ Fourth } & 2900 & May 27-June 18 & $46.7 \pm 4.9$ & $+13.1 \pm 2.2$ & $40.9 \pm 4.1$ & $37.7 \pm 2.3$ & 28.5 & 0.0017 \\
\hline & 3000 & June 9-June 14 & $50.5 \pm 2.1$ & $+14.1 \pm 1.2$ & $41.9 \pm 3.9$ & $37.7 \pm 2.4$ & 29.8 & 0.0009 \\
\hline & 3100 & June 6-June 18 & $49.4 \pm 3.6$ & $+13.8 \pm 1.5$ & $42.8 \pm 3.5$ & $38.2 \pm 2.8$ & 28.4 & 0.0013 \\
\hline & 3200 & June 2-June 20 & $50.9 \pm 3.1$ & $+14.5 \pm 1.5$ & $42.0 \pm 3.6$ & $37.7 \pm 2.3$ & 28.1 & 0.0035 \\
\hline & $4000 \mathrm{a}$ & Oct. 7-Oct. 8 & $36.1 \pm 0.4$ & $+6.9 \pm 0.7$ & $35.7 \pm 2.0$ & $37.7 \pm 1.1$ & 154.8 & 0.0028 \\
\hline & $4000 \mathrm{~b}$ & May 26-June 15 & $49.9 \pm 3.4$ & $+13.1 \pm 1.6$ & $38.6 \pm 2.9$ & $37.1 \pm 1.8$ & 26.0 & 0.0042 \\
\hline \multirow[t]{7}{*}{ Fifth } & 2900 & July $20-$ Sep. 28 & $355.7 \pm 8.9$ & $-11.7 \pm 2.1$ & $34.0 \pm 2.5$ & $38.8 \pm 0.6$ & 161.5 & 0.0080 \\
\hline & 3000 & July 24-Sep. 8 & $342.8 \pm 7.5$ & $-13.9 \pm 1.9$ & $37.8 \pm 2.8$ & $38.4 \pm 1.0$ & 159.2 & 0.0219 \\
\hline & 3100 & July $21-$ Aug. 29 & $341.9 \pm 5.6$ & $-15.1 \pm 1.9$ & $36.4 \pm 1.8$ & $37.4 \pm 1.3$ & 154.5 & 0.0265 \\
\hline & 3200 & July 17-Sep. 11 & $316.0 \pm 99.1$ & $-13.0 \pm 2.3$ & $35.8 \pm 2.7$ & $38.4 \pm 1.2$ & 169.8 & 0.0078 \\
\hline & 4000 & July 13-Aug. 27 & $337.7 \pm 8.1$ & $-16.0 \pm 2.1$ & $39.6 \pm 3.3$ & $37.8 \pm 1.5$ & 152.5 & 0.0066 \\
\hline & $\mathrm{F}$ & Jul. 25-Aug. 16 & $343.4 \pm 4.2$ & $-15.1 \pm 1.1$ & $40.0 \pm 1.6$ & $37.7 \pm 1.2$ & & 36 \\
\hline & $\mathrm{V}$ & July $25-$ Aug. 15 & $341.8 \pm 4.1$ & $-16.1 \pm 1.2$ & $39.7 \pm 1.8$ & $36.9 \pm 1.5$ & & 385 \\
\hline \multirow[t]{5}{*}{ Sixth } & 2900 & Dec. $18-$ Dec. 20 & $120.0 \pm 5.0$ & $-51.9 \pm 0.3$ & $36.5 \pm 1.6$ & $39.4 \pm 0.1$ & 99.4 & 0.0005 \\
\hline & 3000 & Dec. 16-Dec. 20 & $131.0 \pm 10.9$ & $-51.8 \pm 0.3$ & $40.3 \pm 3.5$ & $39.3 \pm 0.2$ & 95.2 & 0.0004 \\
\hline & 3100 & Dec. $14-D e c . ~ 21$ & $125.9 \pm 13.9$ & $-52.0 \pm 1.5$ & $38.3 \pm 4.0$ & $39.3 \pm 0.3$ & 97.0 & 0.0025 \\
\hline & 3200 & Dec. 15-Dec. 21 & $126.3 \pm 12.9$ & $-51.8 \pm 1.5$ & $38.4 \pm 3.9$ & $39.3 \pm 0.3$ & 97.5 & 0.0019 \\
\hline & 4000 & Dec. 22-Jan. 1 & $137.8 \pm 5.4$ & $-51.2 \pm 0.9$ & $42.3 \pm 1.5$ & $39.3 \pm 0.1$ & 96.6 & 0.0054 \\
\hline Seventh & 4000 & July 12-July 16 & $89.1 \pm 0.6$ & $+30.3 \pm 0.7$ & $37.4 \pm 1.6$ & $37.8 \pm 0.9$ & 22.2 & 0.0078 \\
\hline Eighth & 4000 & July $12-J u l y ~ 13$ & $89.7 \pm 0.1$ & $+18.3 \pm 0.9$ & $37.5 \pm 0.1$ & $38.5 \pm 0.0$ & 18.9 & 0.0007 \\
\hline
\end{tabular}

Notes. $t_{\text {evol }}$ - period of the subsequent orbital evolution (the theoretical stream was modeled before this time); D.B. - database from which the observed filament counterpart was selected ( $\mathrm{F}$ - photographic, $\mathrm{V}$ - video-meteor); $t_{\mathrm{act}}$ - typical period of the shower activity during a year; $\alpha_{\mathrm{g}}$ and $\delta_{\mathrm{g}}$ - equatorial coordinates of geocentric radiant; $V_{\mathrm{g}}$ and $V_{\mathrm{h}}$ - geocentric and heliocentric velocity; $\gamma$ - angular distance of the mean radiant from the Sun at the time of the assumed maximum activity of the corresponding shower; $A$ - amount of particles of the whole modeled stream that constitutes the given shower; $N_{\text {sel. }}-$ the number of real meteors selected from the given database. Time $t_{\text {evol }}$ is given in years, the angular quantities are given in degrees, velocities in $\mathrm{km} \mathrm{s}^{-1}$. 
Table 2. Mean orbital characteristics with the dispersion (characterized by standard deviation) of the predicted meteor showers associated with the considered parent body.

\begin{tabular}{|c|c|c|c|c|c|c|c|}
\hline Shower & $t_{\mathrm{evol}}$ or D.B. & $q$ & $a$ & $e$ & $\omega$ & $\Omega$ & $i$ \\
\hline \multirow[t]{6}{*}{ First } & 2900 & $0.290 \pm 0.079$ & $3.775 \pm 0.216$ & $0.924 \pm 0.017$ & $59.7 \pm 12.2$ & $41.3 \pm 11.1$ & $14.3 \pm 3.0$ \\
\hline & 3000 & $0.110 \pm 0.034$ & $3.290 \pm 0.105$ & $0.967 \pm 0.009$ & $36.8 \pm 7.3$ & $60.7 \pm 6.9$ & $16.6 \pm 2.7$ \\
\hline & 3100 & $0.253 \pm 0.075$ & $3.660 \pm 0.219$ & $0.932 \pm 0.016$ & $57.4 \pm 11.6$ & $42.8 \pm 10.6$ & $14.7 \pm 2.3$ \\
\hline & 3200 & $0.182 \pm 0.094$ & $3.465 \pm 0.271$ & $0.949 \pm 0.023$ & $45.0 \pm 15.3$ & $53.5 \pm 13.5$ & $15.6 \pm 3.6$ \\
\hline & 4000 & $0.129 \pm 0.079$ & $3.424 \pm 0.225$ & $0.963 \pm 0.021$ & $37.6 \pm 13.9$ & $61.5 \pm 12.6$ & $19.7 \pm 5.3$ \\
\hline & $\mathrm{R}$ & $0.087 \pm 0.028$ & $2.352 \pm 3.674$ & $0.945 \pm 0.032$ & $27.7 \pm 5.4$ & $78.2 \pm 4.7$ & $23.4 \pm 4.6$ \\
\hline \multirow[t]{8}{*}{ Second } & 2900 & $0.046 \pm 0.023$ & $2.880 \pm 0.112$ & $0.984 \pm 0.007$ & $336.8 \pm 8.9$ & $125.3 \pm 9.2$ & $20.4 \pm 3.7$ \\
\hline & 3000 & $0.041 \pm 0.015$ & $2.863 \pm 0.071$ & $0.986 \pm 0.005$ & $340.4 \pm 3.1$ & $122.3 \pm 4.5$ & $22.1 \pm 2.8$ \\
\hline & 3100 & $0.060 \pm 0.031$ & $2.932 \pm 0.105$ & $0.980 \pm 0.009$ & $336.4 \pm 7.2$ & $126.4 \pm 9.9$ & $20.3 \pm 3.6$ \\
\hline & 3200 & $0.054 \pm 0.040$ & $2.912 \pm 0.201$ & $0.982 \pm 0.011$ & $335.0 \pm 14.9$ & $128.0 \pm 15.6$ & $18.4 \pm 7.1$ \\
\hline & 4000 & $0.067 \pm 0.031$ & $2.937 \pm 0.199$ & $0.977 \pm 0.011$ & $331.2 \pm 9.4$ & $132.4 \pm 14.1$ & $17.1 \pm 4.3$ \\
\hline & $\mathrm{F}$ & $0.084 \pm 0.024$ & $2.628 \pm 0.779$ & $0.963 \pm 0.023$ & $330.6 \pm 4.2$ & $136.2 \pm 4.8$ & $21.3 \pm 3.2$ \\
\hline & V & $0.087 \pm 0.015$ & $2.266 \pm 0.467$ & $0.960 \pm 0.012$ & $330.3 \pm 2.9$ & $139.4 \pm 3.4$ & $22.5 \pm 2.2$ \\
\hline & $\mathrm{R}$ & $0.098 \pm 0.025$ & $1.522 \pm 0.664$ & $0.923 \pm 0.036$ & $331.9 \pm 4.5$ & $128.1 \pm 4.7$ & $21.6 \pm 2.1$ \\
\hline \multirow[t]{12}{*}{ Third } & 500 & $0.978 \pm 0.031$ & $3.019 \pm 0.036$ & $0.676 \pm 0.009$ & $172.5 \pm 2.0$ & $283.2 \pm 0.2$ & $72.2 \pm 0.6$ \\
\hline & 1000 & $0.979 \pm 0.029$ & $3.020 \pm 0.054$ & $0.676 \pm 0.010$ & $173.4 \pm 2.9$ & $283.1 \pm 0.2$ & $71.6 \pm 1.2$ \\
\hline & 1300 & $0.976 \pm 0.030$ & $3.020 \pm 0.059$ & $0.677 \pm 0.010$ & $173.9 \pm 3.9$ & $283.2 \pm 0.2$ & $71.4 \pm 1.4$ \\
\hline & 2000 & $0.984 \pm 0.028$ & $3.027 \pm 0.045$ & $0.675 \pm 0.010$ & $176.6 \pm 4.1$ & $282.9 \pm 0.6$ & $71.4 \pm 1.4$ \\
\hline & 2900 & $0.968 \pm 0.031$ & $3.186 \pm 0.140$ & $0.696 \pm 0.018$ & $170.6 \pm 8.7$ & $280.8 \pm 2.3$ & $68.8 \pm 3.2$ \\
\hline & 3000 & $0.976 \pm 0.030$ & $3.103 \pm 0.088$ & $0.685 \pm 0.013$ & $174.0 \pm 7.7$ & $282.5 \pm 1.8$ & $70.2 \pm 1.9$ \\
\hline & 3100 & $0.968 \pm 0.029$ & $3.190 \pm 0.145$ & $0.696 \pm 0.018$ & $170.2 \pm 8.5$ & $281.2 \pm 2.6$ & $68.8 \pm 3.4$ \\
\hline & 3200 & $0.984 \pm 0.028$ & $3.085 \pm 0.050$ & $0.681 \pm 0.010$ & $176.9 \pm 7.6$ & $282.7 \pm 1.7$ & $70.5 \pm 1.3$ \\
\hline & 4000 & $0.984 \pm 0.030$ & $3.080 \pm 0.069$ & $0.680 \pm 0.011$ & $176.4 \pm 8.1$ & $283.2 \pm 2.3$ & $68.2 \pm 3.0$ \\
\hline & $\mathrm{F}$ & $0.978 \pm 0.004$ & $3.008 \pm 0.508$ & $0.667 \pm 0.050$ & $170.8 \pm 3.5$ & $283.3 \pm 0.8$ & $71.7 \pm 1.6$ \\
\hline & V & $0.979 \pm 0.003$ & $2.597 \pm 0.222$ & $0.621 \pm 0.032$ & $171.4 \pm 3.1$ & $283.2 \pm 0.6$ & $70.8 \pm 1.4$ \\
\hline & $\mathrm{R}$ & $0.973 \pm 0.005$ & $2.979 \pm 0.324$ & $0.669 \pm 0.035$ & $167.5 \pm 2.8$ & $282.9 \pm 0.9$ & $70.8 \pm 1.6$ \\
\hline \multirow[t]{7}{*}{ Fourth } & 2900 & $0.069 \pm 0.043$ & $3.002 \pm 0.170$ & $0.978 \pm 0.012$ & $206.5 \pm 9.1$ & $256.2 \pm 8.2$ & $17.1 \pm 3.9$ \\
\hline & 3000 & $0.053 \pm 0.020$ & $2.886 \pm 0.068$ & $0.982 \pm 0.006$ & $203.7 \pm 4.5$ & $261.3 \pm 4.7$ & $19.2 \pm 2.1$ \\
\hline & 3100 & $0.054 \pm 0.021$ & $2.909 \pm 0.108$ & $0.982 \pm 0.007$ & $205.6 \pm 7.8$ & $258.8 \pm 7.3$ & $19.3 \pm 4.3$ \\
\hline & 3200 & $0.053 \pm 0.025$ & $2.903 \pm 0.102$ & $0.982 \pm 0.008$ & $205.2 \pm 7.8$ & $260.1 \pm 8.1$ & $18.4 \pm 5.0$ \\
\hline & $4000 \mathrm{a}$ & $0.155 \pm 0.004$ & $3.323 \pm 0.005$ & $0.953 \pm 0.001$ & $139.9 \pm 5.0$ & $11.8 \pm 5.0$ & $16.3 \pm 1.2$ \\
\hline & $4000 \mathrm{~b}$ & $0.095 \pm 0.029$ & $2.889 \pm 0.150$ & $0.967 \pm 0.010$ & $212.5 \pm 7.4$ & $256.5 \pm 7.3$ & $16.4 \pm 3.3$ \\
\hline & $\mathrm{R}$ & $0.086 \pm 0.022$ & $1.749 \pm 1.084$ & $0.937 \pm 0.031$ & $206.5 \pm 5.6$ & $258.4 \pm 6.8$ & $22.0 \pm 2.4$ \\
\hline \multirow[t]{8}{*}{ Fifth } & 2900 & $0.263 \pm 0.083$ & $3.684 \pm 0.227$ & $0.930 \pm 0.018$ & $123.1 \pm 12.0$ & $335.2 \pm 14.2$ & $14.9 \pm 2.2$ \\
\hline & 3000 & $0.145 \pm 0.060$ & $3.384 \pm 0.168$ & $0.958 \pm 0.015$ & $136.9 \pm 10.0$ & $318.9 \pm 11.9$ & $15.5 \pm 2.7$ \\
\hline & 3100 & $0.143 \pm 0.040$ & $3.327 \pm 0.127$ & $0.957 \pm 0.010$ & $141.8 \pm 8.8$ & $313.0 \pm 10.6$ & $17.8 \pm 3.3$ \\
\hline & 3200 & $0.199 \pm 0.080$ & $3.504 \pm 0.216$ & $0.944 \pm 0.020$ & $132.3 \pm 12.6$ & $324.2 \pm 14.9$ & $15.3 \pm 2.9$ \\
\hline & 4000 & $0.100 \pm 0.061$ & $3.308 \pm 0.172$ & $0.970 \pm 0.017$ & $148.3 \pm 11.1$ & $306.6 \pm 12.7$ & $21.7 \pm 4.7$ \\
\hline & $\mathrm{F}$ & $0.093 \pm 0.022$ & $3.0 \pm 1.1$ & $0.966 \pm 0.013$ & $148.2 \pm 4.0$ & $312.0 \pm 5.2$ & $24.7 \pm 3.2$ \\
\hline & V & $0.087 \pm 0.021$ & $2.692 \pm 4.2$ & $0.962 \pm 0.016$ & $150.0 \pm 3.8$ & $308.9 \pm 4.7$ & $26.4 \pm 3.3$ \\
\hline & $\mathrm{R}$ & $0.077 \pm 0.020$ & $1.950 \pm 0.7$ & $0.953 \pm 0.025$ & $153.7 \pm 3.8$ & $306.2 \pm 2.9$ & $27.8 \pm 3.3$ \\
\hline \multirow[t]{5}{*}{ Sixth } & 2900 & $0.970 \pm 0.011$ & $3.643 \pm 0.130$ & $0.733 \pm 0.012$ & $20.9 \pm 2.7$ & $87.4 \pm 0.6$ & $61.3 \pm 3.6$ \\
\hline & 3000 & $0.956 \pm 0.010$ & $3.377 \pm 0.247$ & $0.716 \pm 0.023$ & $11.6 \pm 9.5$ & $86.6 \pm 1.7$ & $69.3 \pm 7.7$ \\
\hline & 3100 & $0.965 \pm 0.024$ & $3.502 \pm 0.316$ & $0.722 \pm 0.027$ & $14.1 \pm 11.8$ & $86.5 \pm 2.1$ & $65.1 \pm 8.7$ \\
\hline & 3200 & $0.966 \pm 0.029$ & $3.499 \pm 0.296$ & $0.722 \pm 0.028$ & $15.3 \pm 10.7$ & $87.7 \pm 4.0$ & $65.4 \pm 8.6$ \\
\hline & 4000 & $0.964 \pm 0.029$ & $3.378 \pm 0.120$ & $0.714 \pm 0.013$ & $17.5 \pm 3.6$ & $94.9 \pm 2.5$ & $73.6 \pm 3.4$ \\
\hline Seventh & 4000 & $0.140 \pm 0.008$ & $3.308 \pm 0.011$ & $0.958 \pm 0.002$ & $41.4 \pm 5.3$ & $110.4 \pm 5.3$ & $17.5 \pm 1.8$ \\
\hline Eighth & 4000 & $0.160 \pm 0.006$ & $3.343 \pm 0.011$ & $0.952 \pm 0.002$ & $226.0 \pm 9.5$ & $287.9 \pm 9.9$ & $12.1 \pm 2.0$ \\
\hline
\end{tabular}

Notes. $t_{\text {evol }}$ - as in Table 1; D.B. - database from which the observed counterpart of filament was selected (F-photographic; V - video-meteor; R - radio-meteor database); $q$ - perihelion distance; $a$ - semi-major axis; $e$ - eccentricity; $\omega$ - argument of perihelion; $\Omega$ - longitude of ascending node; and $i$-inclination to the ecliptic. Quantities $q$ and $a$ are given in astronomical units and angular elements in degrees. 